\title{
THE ABSOLUTE OPTICAL INSTRUMENT*
}

\author{
BY
}

J. L. SYNGE

1. Introduction. An absolute optical instrument is a system of transparent media which gives a precise point-image of each object-point lying in a threedimensional region, the law of light propagation being that of geometrical optics. For a long time only two absolute instruments were known, the plane mirror (or combination of plane mirrors) and the "fish's eye" of Maxwell, $\dagger$ which consists of a single isotropic medium of variable refractive index

$$
n=n_{0} a^{2} /\left(a^{2}+r^{2}\right),
$$

where $n_{0}, a$ are constants, and $r$ is the distance from a fixed point. Maxwell's medium has been generalized by Lenz, $\ddagger$ and Boegehold and Herzberger $\S$ have given an infinite class of absolute instruments, consisting of homogeneous isotropic media bounded by concentric spheres.

Less attention has been paid to the question of the realization of an absolute instrument by a distribution of transparent media than to the relations between object and image which must hold if the instrument is absolute. In the simplest case, in which the initial and final media are homogeneous and isotropic, it has been shown in various ways by Maxwell, Bruns, Klein, and Liebmann $\|$ that if $A, B$ are the images of object-points $A^{\prime}, B^{\prime}$, then

$$
n^{\prime} A^{\prime} B^{\prime}=n A B,
$$

where $n^{\prime}, n$ are the (constant) refractive indices of the initial and final media. This relation may also be written

$$
\left[A^{\prime} B^{\prime}\right]=[A B]
$$

where the brackets indicate optical lengths.

* Presented to the Society, March 27, 1937; received by the editors February 26, 1937 and June $16,1937$.

$\dagger$ J. C. Maxwell, Cambridge and Dublin Mathematical Journal, vol. 8 (1854), p. 188; Scientific Papers, vol. 1, Cambridge, 1890, pp. 76-79.

$\ddagger$ W. Lenz, Probleme der Moderne Physik, edited by P. Debye, Leipzig, 1928, pp. 198-207.

$\S \mathrm{H}$. Boegehold and M. Herzberger, Zeitschrift für Angewandte Mathematik und Mechanik, vol. 15 (1935), pp. 157-178.

|| For references and an historical account by H. Boegehold, see Czapski-Eppenstein, Grundz üge der Theorie der Optischen Instrumente, Leipzig, 1924, p. 213 et seq. See also Carathéodory, Sitzungsberichte der Bayerischen Akademie der Wissenschaften, Mathematisch-Physikalische Klasse, 1926, pp. 1-18. 
Carathéodory* has extended this result to the case of more general media, namely, to those in which the velocity of propagation of light is the same in opposite directions and this velocity, considered as a function of the direction of a ray, satisfies certain conditions of analyticity. $\dagger$ Further, by introducing the idea of the field of the instrument, he made his result applicable to actual instruments.

In the present paper the approach to the problem of the absolute instrument differs from that of Carathéodory. Attention is directed to the surface of components rather than the wave-surface, and we are thereby enabled to see that when the optical character of one of the terminal media is assigned, the optical character of the other is rather closely restricted if the instrument is absolute.

Extension to the case where the space is Riemannian and of $N$ dimensions may easily be made; but since the problem is one of considerable optical interest, it has been thought best not to complicate the presentation by this extension. The problem may also be presented as a problem in Finsler space.

2. Fundamental theory. Hamilton's great achievement in geometrical optics was the reconciliation, in the form of a single mathematical treatment, of the emission theory of light and the wave theory, $\ddagger$ or, in the language of pure mathematics, the calculus of variations and the theory of contact transformations. This comprehensive view places at our disposal for the discussion of any problem in geometrical optics two alternative methods-the raymethod and the wave-method.

The methods employed in the present paper are essentially those of Hamilton, although for brevity an indicial notation will be used, suffixes having the range 1, 2, 3, with the usual summation convention.§ Except for a modification of one of Hamilton's definitions to suit present purposes, there is nothing in the present paper which might not have been given by Hamilton a century ago as an immediate deduction from his theory. The dis-

${ }^{*}$ C. Carathéodory, loc. cit. The part of this paper containing the fundamental theorem is reproduced, with no essential modification, in M. Born, Optik, Berlin, 1933, pp. 61-63.

$\dagger$ Professor Carathéodory has informed me by letter that in establishing the result he had exclusively in mind media of the Fresnel type, or media in which similar conditions of analyticity are: satisfied. Unfortunately these conditions were not stated in his paper, and the reader might come to the false conclusion that the result follows from the single assumption that the velocity of propagation is the same in opposite directions. That is not so, and it is not difficult to construct artificial examples in illustration. Carathéodory's method is discussed in $\$ 9$.

$\ddagger$ Cf. G. Prange, W. R. Hamiltons Abhandlungen zur Strahlenoptik, Leipzig, 19.33, Anmerkungen, p. 104; The Mathematical Papers of Sir W. R. Hamilton, vol. 1, Cambridge, 1931, pp. 277, 497. The latter will be referred to as $M$. P. H.

§ J. L. Synge, Journal of the Optical Society of America, vol. 27 (1937), pp. 75-82. 
cussion of an absolute instrument does not seem to have occurred to him; the idea arose out of Gaussian optics.

The essential parts of the theory will now be developed, care being taken to present them in a form applicable to an absolute instrument. Certain exceptional features are present in such an instrument which may render invalid statements valid in more general cases.

The instrument is composed of any number of media. Each medium has a positive medium-function $v\left(x_{r}, \alpha_{r}\right)$, a function of rectangular cartesian coordinates $x_{r}$ and of direction cosines $\alpha_{r} ; v$ is homogeneous of degree unity in the direction cosines. It is in general multiple-valued. If the units of space and time are so chosen that the velocity of light in vacuo is unity, then $1 / v$ is the ray velocity in the medium for a ray having direction cosines $\alpha_{r}$ at the point $x_{r}$. (For an isotropic medium, $v$ is equal to the refractive index.)

By Fermat's principle (accepted as a basic hypothesis) rays satisfy the variational principle $\delta \int v d s=0, d s$ being an element of arc.

The components of normal slowness corresponding to a ray through $x_{r}$ with direction cosines $\alpha_{r}$ are defined as

$$
\sigma_{r}=\partial v / \partial \alpha_{r} .
$$

They were so called by Hamilton* because the vector $\sigma_{r}$ stands normal to the wave and its magnitude is equal to the reciprocal of the wave velocity.

The right-hand side of (2.1) being homogeneous of degree zero in the direction cosines, we can eliminate their ratios and obtain the medium-equation

$$
\Omega\left(x_{r}, \sigma_{r}\right)=0 .
$$

If, from an assigned point $x_{r}$, we measure off along each straight line with direction cosines $\alpha_{r}$ a length $1 / v$, obtaining a point with relative coordinates $\alpha_{r} / v$, the surface so obtained is the wave-surface corresponding to $x_{r}$. On the other hand if we draw from $x_{r}$ the totality of vectors $\sigma_{r}$ satisfying (2.2), we get the surface of components corresponding to $x_{r}$. The wave-surface and the surface of components are reciprocal surfaces with respect to the unit sphere with centre $x_{r} . \dagger$

* M.P.H., p. 278; M. Herzberger, Strahlenoptik, Berlin, 1931, p. 9, calls this vector the "normal vector." "Slowness vector" might be a more descriptive abbreviation for the full title namely, "the vector representing the normal slowness of wave-propagation." These components are of course the optical analogues of the generalized momenta $\left(p_{r}\right)$ of Hamilton's dynamical theory.

$\dagger$ It seems a pity to reject Hamilton's very suggestive terminology, wave-surface and surface of components, in favor of the names indicatrix and figuratrix, which carry no intrinsic meaning. Unless the extension to $n$ dimensions appears an important advance, it does not seem historically correct to assign priority in the consideration of these surfaces to Minkowski and Hadamard (cf. C. Carathéodory, Variationsreclinung, Leipzig und Berlin, 1935, p. 247). Hamilton had a priority of seventy years, and even he assigned priority to Cauchy. 
The optical length of any curve $C$ joining points $P, Q$ is

$$
[P Q]=\int_{C} v d s,
$$

the directional arguments of $v$ being the direction cosines of the tangent to $C$, in the sense $P$ to $Q$, and $d s$ being a positive element of arc. Giving a weak variation with, in general, displacements of the end points $P, Q$, we know from the calculus of variations that

$$
\delta[P Q]=\left(\frac{\partial v}{\partial \alpha_{r}} \delta x_{r}\right)_{Q}-\left(\frac{\partial v}{\partial \alpha_{r}} \delta x_{r}\right)_{P}-\int_{C}\left(\frac{d}{d s} \frac{\partial v}{\partial \alpha_{r}}-\frac{\partial v}{\partial x_{r}}\right) \delta x_{r} d s .
$$

Suppose now that $C$ is a ray; then, by the Euler equations, the integral vanishes and we have, in the notation of (2.1),

$$
\delta[P Q]=\left(\sigma_{r} \delta x_{r}\right)_{Q}-\left(\sigma_{r} \delta x_{r}\right)_{P} .
$$

The characteristic function of an instrument is a function $V$ of the coordinates $x_{r}^{\prime}$ of a point $A^{\prime}$ in the initial medium $M^{\prime}$ and the coordinates $x_{r}$ of a point $A$ in the final medium $M$, such that $V$ is equal to the optical length of a ray joining $A^{\prime}$ and $A$. The function $V$ may not be defined for all values of the six variables $x_{r}^{\prime}, x_{r}$ corresponding to points $A^{\prime}, A$ in the initial and final media respectively, because there may exist no ray joining $A^{\prime}$ and $A$. In general $V$ is multiple-valued, since $v$ is multiple-valued.

We shall use accents to denote quantities pertaining to the initial medium $M^{\prime}$ of the instrument; quantities pertaining to the final medium will be left unaccented. Different rectangular axes for $x_{r}^{\prime}$ and $x_{r}$ may be used for $M^{\prime}$ and $M$ respectively. Inspection of the method by which (2.5) has been established shows that the axes to which quantities at $P$ are referred need not be the same as the axes to which quantities at $Q$ are referred. From (2.5) we see at once that if we pass from a ray joining points $A^{\prime}, A$ to an adjacent ray joining points $B^{\prime}, B$, the variation in $V$ is*

$$
\delta V=\sigma_{r} \delta x_{r}-\sigma_{r}^{\prime} \delta x_{r}^{\prime} .
$$

* This is the fundamental equation of Hamilton's theory, called by him the equation of the characteristic function (M.P.H., p. 168). It is of course the optical analogue of the fundamental equation defining contact transformations in dynamics. Hamilton did not frame his theory to take into account the exceptional case presented by an absolute instrument; he assumed that six arbitrary variations $\delta x_{r}^{\prime}, \delta x_{r}$ were permissible in (2.6), so that

$$
\partial V / \partial x_{r}=\sigma_{r}, \quad \partial V / \partial x_{r}{ }^{\prime}=-\sigma_{r}{ }^{\prime} .
$$

This cannot be done in the case of an absolute instrument if the points $x_{r}{ }^{\prime}$ and $x_{r}$ correspond to object: and image respectively. The argument of the present paper, which makes no such illegitimate assumption, has already been given by Herzberger (op. cit., p. 12) in a different notation. 
Following Carathéodory, we shall say that a ray passing through a point $A^{\prime}$ of $M^{\prime}$ with direction cosines $\alpha_{r}^{\prime}$ lies in the field of the instrument if it passes through the instrument to the final medium $M$. At a given point $A^{\prime}$ of $M^{\prime}$ the directions of the rays lying in the field of the instrument will be bounded by a cone of unidirectional straight lines drawn out from $A^{\prime}$.

3. Necessary conditions for an absolute instrument. Consider the rays emanating in the field of the instrument from an initial point $A^{\prime}$. As the medium-functions of the media in the instrument may be multiple-valued, these rays may pass through the final medium in a number of congruences, which we distinguish from one another by calling them congruences of different types.

If all the rays in the final congruence of type $T$ pass through a single point $A$, we may say that the instrument is absolute (type $T$ ) with respect to the source $A^{\prime}, A$ being the image (type $T$ ) of $A^{\prime}$. If the instrument is absolute as above with respect to all initial points and directions in the field of the instrument, we may say that the instrument is absolute of type $T$. If it is absolute for all types, we may say that it is completely absolute.

In the present paper we shall be concerned with absolute character for final congruences of a definite type. For simplicity, we shall refer to an instrument possessing this absolute character as absolute, without further explicit qualification.

In an absolute instrument there exists a one-to-one correspondence

$$
x_{r}^{\prime}=x_{r}^{\prime}\left(x_{s}\right), \quad x_{r}=x_{r}\left(x_{s}^{\prime}\right),
$$

between the points of the initial and final media, $A$ with coordinates $x_{r}$ being the image of $A^{\prime}$ with coordinates $x_{r}^{\prime}$.

If, given $A^{\prime}$, we seek points $A$ such that a ray joins $A^{\prime}$ to $A$, we find our choice of $A$ restricted to that part of the final medium traversed by the congruence of rays from $A^{\prime}$. Thus, given $A^{\prime}$ with coordinates $x_{r}^{\prime}$, the characteristic $V\left(x_{r}^{\prime}, x_{r}\right)$ is defined only for a restricted range of values of $x_{r}$. This range includes of course the image of $A^{\prime}$; the coordinates of that image are, by (3.1), functions of $x_{r}^{\prime}$. When $A$ is chosen at the image of $A^{\prime}$, there is an infinity of rays joining the two points, but by Fermat's principle their optical lengths are all the same. Hence in an absolute instrument there exists an absolute characteristic, namely, a function of the three initial coordinates, or of the three final coordinates, whose value is equal to the optical length of a ray joining object to image. This absolute characteristic will be denoted by $F$.

Let us now take two adjacent image-points $x_{r}, x_{r}+\delta x_{r}$, and compare the optical lengths of the rays drawn to these points from their respective ob- 
jects. The difference between these optical lengths, by (2.6) and (3.1), is given by

$$
\begin{aligned}
\delta F & =\sigma_{r} \delta x_{r}-\sigma_{r}^{\prime} \delta x_{r}^{\prime} \\
& =\sigma_{r} \delta x_{r}-\sigma_{s}^{\prime} \frac{\partial x_{s}^{\prime}}{\partial x_{r}} \delta x_{r},
\end{aligned}
$$

where $\sigma_{r}^{\prime}$ are the components of any ray (lying in the field) through the object $x_{r}^{\prime}$ and $\sigma_{r}$ the components of the corresponding ray through the image $x_{r}$. Since $\delta x_{r}$ are arbitrary, (3.2) gives the three equations

$$
\frac{\partial F}{\partial x_{r}}=\sigma_{r}-\sigma_{s}^{\prime} \frac{\partial x_{s}^{\prime}}{\partial x_{r}}
$$

which are the fundamental equations of the present paper.

The implications of (3.3) may be explored in various ways. Thus we may suppose the absolute characteristic $F$ and the transformation (3.1) given, and inquire into the conditions imposed by (3.3) on the optical characters of the initial and final media. Or we may suppose that the initial and final media are of assigned optical characters, given by medium-functions $v^{\prime}\left(x_{r}{ }^{\prime}, \alpha_{r}^{\prime}\right)$, $v\left(x_{r}, \alpha_{r}\right)$ or by surfaces of components

$$
\Omega^{\prime}\left(x_{r}^{\prime}, \sigma_{r}^{\prime}\right)=0, \quad \Omega\left(x_{r}, \sigma_{r}\right)=0 ;
$$

we may then inquire into the conditions imposed by (3.3) on the absolute characteristic $F$ and the transformation (3.1). The latter type of approach has been the usual one.

4. The optical character of one terminal medium in an absolute instrument deduced from that of the other. Let us write (3.3) as

$$
\sigma_{r}=a_{r s} \sigma_{s}^{\prime}+b_{r}
$$

where

$$
a_{r s}=\frac{\partial x_{s}^{\prime}}{\partial x_{r}}, \quad b_{r}=\frac{\partial F}{\partial x_{r}} .
$$

Let us consider (4.1) as a transformation expressing final components $\sigma_{r}$ in terms of initial components $\sigma_{r}^{\prime}$, the corresponding object and image-points $\left(A^{\prime}\right.$ and $A$ ) and the absolute characteristic being given. Thus for present purposes $a_{r s}$ and $b_{r}$ are to be treated as constants; (4.1) is a linear transformation.

Conisider now the surface of components $S^{\prime}$ corresponding to $A^{\prime}$ (with equation $\Omega^{\prime}=0$ ) and the surface of components $S$ corresponding to $A$ (with 
equation $\Omega=0$ ). Consider any ray through $A^{\prime}$ in the field of the instrument. Let its components be $\sigma_{r}^{\prime}$. Then the vector drawn from $A^{\prime}$ with components $\sigma_{r}^{\prime}$ parallel to the axes of $x_{r}^{\prime}$ has its end on $S^{\prime}$. The corresponding final ray has components given by (4.1). But the vector drawn from $A$ with compo-

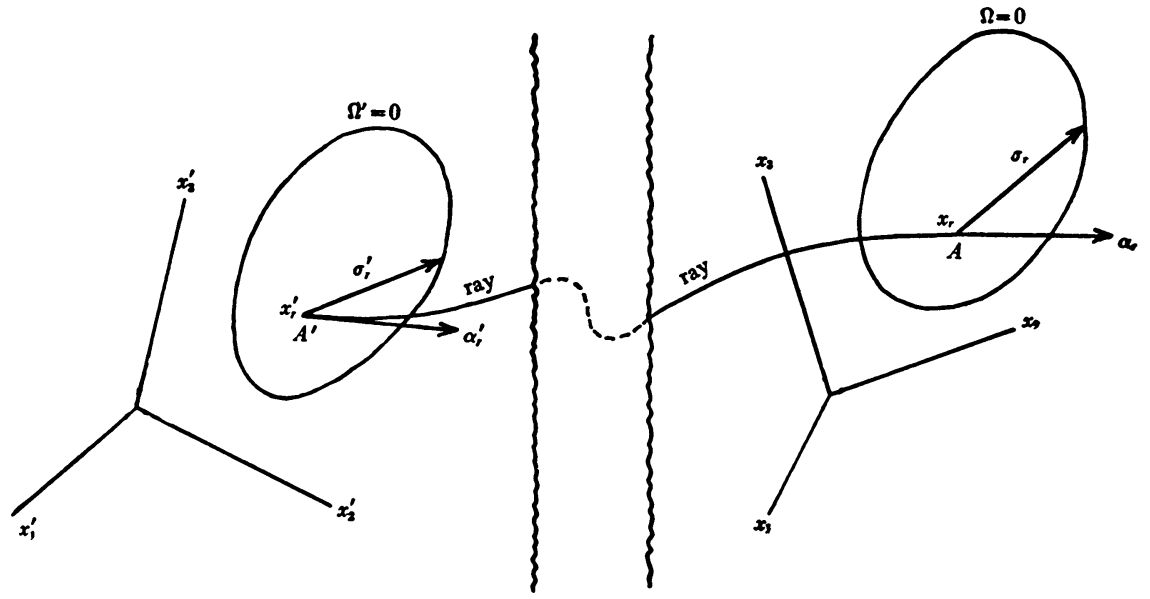

Fig. 1

nents $\sigma_{r}$ parallel to the axes of $x_{r}$ must have its end on $S$ (Fig. 1). Hence we have the following result:

THEOREM I. In an absolute instrument the portions lying in the field of the instrument of the surfaces of components corresponding to an object-point $A^{\prime}$ and its image $A$ are transformable into one another by a linear transformation, whose coefficients are in general functions of the coordinates of $A^{\prime}$.

Thus, insofar as the condition (3.3) is concerned, we may choose arbitrarily the following:

(i) the absolute characteristic $F$,

(ii) the correspondence $x_{r}=x_{r}\left(x_{s}^{\prime}\right)$,

(iii) the surfaces of components (that is, the equation $\Omega^{\prime}\left(x_{r}^{\prime}, \sigma_{r}^{\prime}\right)=0$ ) throughout the initial medium.

Then the surfaces of components for the final medium (and hence its medium-function $v$ ) can be found to satisfy (3.3). We have in fact by (3.3)

$$
\sigma_{r}^{\prime}=\sigma_{s} \frac{\partial x_{s}}{\partial x_{r}^{\prime}}-\frac{\partial F}{\partial x_{r}^{\prime}}
$$

so that $\Omega^{\prime}\left(x_{r}^{\prime}, \sigma_{r}^{\prime}\right)=0$ implies

$$
\Omega^{\prime}\left(x_{r}^{\prime}\left(x_{s}\right), \sigma_{s} \frac{\partial x_{s}}{\partial x_{r}^{\prime}}-\frac{\partial F}{\partial x_{r}^{\prime}}\right)=0 ;
$$


these are the required surfaces of components in the final medium, (4.4) being immediately expressible in the form $\Omega\left(x_{r}, \sigma_{r}\right)=0$.

In particular we may observe that if there is an absolute instrument in which the surfaces of components in one of the terminal media are ellipsoids (the wave-surfaces being consequently, by the reciprocal relation, ellipsoids also), then the surfaces of components in the other terminal medium are also ellipsoids (and so are the wave-surfaces).

More generally, if in an absolute instrument the surfaces of components throughout one of the terminal media are undegenerate algebraic surfaces of the $n$th degree, then the surfaces of components throughout the other terminal medium are also algebraic surfaces of the $n$th degree.

The assumption of algebraic character carries with it an important implication (as would also a suitable assumption regarding analyticity), namely, that a linear transformation which carries the portion of one of the surfaces of components corresponding to rays lying in the field of the instrument into a portion of the other surface of components, also carries the whole of one surface of components into the whole of the other. It is this fact that enables us to make statements about complete surfaces of components, although our data deal only with the portions corresponding to rays lying in the field of the instrument.

5. The case where the surfaces of components in the terminal media are general algebraic surfaces. Let us now suppose that there is an instrument with given terminal media in which the surfaces of components are undegenerate algebraic surfaces of the $n$th degree. We may write

$$
\begin{aligned}
& \Omega^{\prime} \equiv 1+c_{r}^{\prime} \sigma_{r}^{\prime}+c_{r s}^{\prime} \sigma_{r}^{\prime} \sigma_{s}^{\prime}+\cdots(\text { to } n+1 \text { terms })=0,\left(c_{r s}^{\prime}=c_{s r}^{\prime}\right), \\
& \Omega \equiv 1+c_{r} \sigma_{r}+c_{r s} \sigma_{r} \sigma_{s}+\cdots(\text { to } n+1 \text { terms })=0,\left(c_{r s}=c_{s r}\right),
\end{aligned}
$$

where $c_{r}^{\prime}, c_{r s}^{\prime}, \cdots$ are assigned functions of $x_{r}^{\prime}$, and $c_{r}, c_{r s}, \cdots$ are assigned functions of $x_{r}$.

We wish to investigate the possibility of constructing an absolute instrument with these terminal media, taking into consideration the condition (3.3).

Adopting the notation of (4.1) and substituting in (5.2), we get

$$
\Omega \equiv 1+c_{r}\left(a_{r s} \sigma_{s}^{\prime}+b_{r}\right)+c_{r s}\left(a_{r t} \sigma_{t}^{\prime}+b_{r}\right)\left(a_{s u} \sigma_{u}^{\prime}+b_{s}\right)+\cdots=0 ;
$$

this must be the same as the surface (5.1). Thus we require

$$
\begin{aligned}
1+c_{r} b_{r}+c_{r s} b_{r} b_{s}+\cdots & =\theta, \\
c_{s} a_{s r}+2 c_{s t} a_{s r} b_{t}+\cdots & =\theta c_{r}^{\prime},
\end{aligned}
$$


where $\theta$ is a factor of proportionality. Here we have a set of equations for five unknown functions of $x_{r}$, namely, $F, x_{r}^{\prime}, \theta$. Omitting from consideration for obvious reasons the case $n=1$, let us pass to the case $n=2$, for which the surfaces are quadrics. There are then ten coefficients in $\Omega$ and hence ten equations in (5.4) for five unknowns. If $n>2$, there are of course more equations. It is therefore in general impossible to construct an absolute instrument with arbitrarily assigned terminal media in which the surfaces of components (or the wavesurfaces) are undegenerate algebraic surfaces of the second degree or of higher degree.

6. The case where the surfaces of components in the terminal media are central algebraic surfaces. By a central surface we shall understand a surface which has a centre at the origin; for a surface of components or wave-surface, the origin is the point in the medium to which the surface corresponds.

A linear transformation (4.1) which carries a central surface into a central surface must lack the absolute term. Hence we have this result (of importance in connection with Carathéodory's theorem and implicit in his proof, unless replaced by a condition of analyticity):

THEOREM II. In an absolute optical instrument in which the surfaces of components (or, equivalently, the wave-surfaces) are undegenerate central algebraic surfaces, the absolute characteristic is a constant. This result also holds for degenerate central surfaces, provided that each of the separate surfaces given by degeneration is central.

Under these circumstances the transformation of components is simply

$$
\sigma_{r}=\sigma_{s}^{\prime} \frac{\partial x_{s}^{\prime}}{\partial x_{r}}
$$

7. The case of isotropic terminal media. When the terminal media are isotropic, the surfaces of components are spheres with the equations

$$
\begin{aligned}
& \Omega^{\prime} \equiv \sigma_{r}^{\prime} \sigma_{r}^{\prime}-n^{\prime 2}=0, \\
& \Omega \equiv \sigma_{r} \sigma_{r}-n^{2}=0,
\end{aligned}
$$

where $n^{\prime}, n$ are refractive indices, functions of position. Applying (6.1), we see that if the instrument is absolute, the correspondence of object and imagepoints must satisfy

$$
\frac{\partial x_{s}^{\prime}}{\partial x_{r}} \frac{\partial x_{t}^{\prime}}{\partial x_{r}}=\frac{n^{2}}{n^{\prime 2}} \delta_{s t},
$$

where $\delta_{s t}$ is the Kronecker delta. These partial differential equations, six in 
number, are not in general soluble for the three unknowns $x_{r}{ }^{\prime}$. We deduce from them

$$
\frac{\partial x_{s}^{\prime}}{\partial x_{r}}=\frac{n^{2}}{n^{\prime 2}} \frac{\partial x_{r}}{\partial x_{s}^{\prime}}
$$

and hence

$$
\begin{aligned}
n^{2} d s^{2}=n^{2} d x_{r} d x_{r} & =n^{2} \frac{\partial x_{r}}{\partial x_{s}^{\prime}} \frac{\partial x_{r}}{\partial x_{t}^{\prime}} d x_{s}^{\prime} d x_{t}^{\prime} \\
& =\frac{n^{\prime 4}}{n^{2}} \frac{\partial x_{s}^{\prime}}{\partial x_{r}} \frac{\partial x_{t}^{\prime}}{\partial x_{r}} d x_{s}^{\prime} d x_{t}^{\prime} \\
& =n^{\prime 2} d x_{s}^{\prime} d x_{s}^{\prime} \\
& =n^{\prime 2} d s^{\prime 2} .
\end{aligned}
$$

This establishes (by a method different from, and perhaps more direct than, the method of Caratheodory) the equality of optical lengths for object and image elements in an absolute instrument for which the terminal media are isotropic and in general heterogeneous. A necessary condition for the absolute character of such an instrument is the applicability of the terminal media, considered as Riemannian spaces with metrics $n d s, n^{\prime} d s^{\prime}$.

8. The case of Fresnel media. In a biaxial crystal, or homogeneous Fresnel medium, the surface of components referred to principal axes has the equation*

$$
\frac{\sigma_{1}{ }^{2}}{1-c_{1}{ }^{2} q^{2}}+\frac{\sigma_{2}{ }^{2}}{1-c_{2}{ }^{2} q^{2}}+\frac{\sigma_{3}{ }^{2}}{1-c_{3}{ }^{2} q^{2}}=0, \quad q^{2} \equiv \sigma_{r} \sigma_{r},
$$

where $c_{r}$ are constants (the principal velocities). Cleared of fractions, this equation reads

$$
q^{2} A_{m n}^{0} \sigma_{m} \sigma_{n}-B_{m n}^{0} \sigma_{m} \sigma_{n}+1=0, \quad q^{2} \equiv \sigma_{r} \sigma_{r}
$$

where

$$
\left\{\begin{array}{lll}
A_{11}^{0}=c_{2}{ }^{2} c_{3}{ }^{2}, & A_{22}^{0}=c_{3}{ }^{2} c_{1}{ }^{2}, & A_{33}^{0}=c_{1}{ }^{2} c_{2}{ }^{2}, \\
B_{11}^{0}=c_{2}{ }^{2}+c_{3}{ }^{2}, & B_{22}^{0}=c_{3}{ }^{2}+c_{1}{ }^{2}, & B_{33}^{0}=c_{1}{ }^{2}+c_{2}{ }^{2},
\end{array}\right.
$$

and $A_{m n}^{0}=B_{m n}^{0}=0$ if $m \neq n$.

If we change to an arbitrary set of rectangular axes (not principal) the surface of components has an equation of the form

$$
q^{2} A_{m n} \sigma_{m} \sigma_{n}-B_{m n} \sigma_{m} \sigma_{n}+1=0, \quad q^{2} \equiv \sigma_{r} \sigma_{r},
$$

* Cf. M.P.H., p. 280; Born, op. cit., p. 224. 
where $A_{m n}=A_{n m}, B_{m n}=B_{n m}$, these quantities being found from $A_{m n}^{0}, B_{m n}^{0}$ respectively by application of the rules of tensor transformation. Given an equation (8.4), necessary and sufficient conditions that it should define the surface of components in a homogeneous Fresnel medium are as follows:

(i) The roots of the determinantal equations

$$
\left|A_{m n}-\lambda \delta_{m n}\right|=0, \quad\left|B_{m n}-\mu \delta_{m n}\right|=0,
$$

are of the form

$$
\left\{\begin{array}{lll}
\lambda_{1}=c_{2}{ }^{2} c_{3}^{2}, & \lambda_{2}=c_{3}{ }^{2} c_{1}^{2}, & \lambda_{3}=c_{1}{ }^{2} c_{2}{ }^{2}, \\
\mu_{1}=c_{2}{ }^{2}+c_{3}{ }^{2}, & \mu_{2}=c_{3}{ }^{2}+c_{1}{ }^{2}, & \mu_{3}=c_{1}{ }^{2}+c_{2}{ }^{2},
\end{array}\right.
$$

where the $c_{r}$ are constants.

(ii) Each of the three perpendicular directions defined by

$$
A_{m n} \sigma_{n}-\lambda_{r} \sigma_{m}=0
$$

for fixed $r$, coincides with the direction defined by

$$
B_{m n} \sigma_{n}-\mu_{r} \sigma_{m}=0
$$

for the same value of $r$.

A heterogeneous Fresnel medium may be defined as a medium in which at each point there exists a set of axes such that the surface of components corresponding to that point has the form (8.1). Equivalently, we may say that, for a single set of rectangular cartesian coordinates throughout the medium, a heterogeneous Fresnel medium is one in which the surface of components at any point has the form (8.4), where $A_{m n}, B_{m n}$ are functions of the coordinates $x_{r}$ satisfying the conditions (i) and (ii) above, in which $c_{r}$ are no longer constants but functions of $x_{r}$. We shall confine our attention to the undegenerate case in which $c_{1}, c_{2}, c_{3}$ are distinct.

Let us now consider an absolute instrument in which the terminal media are Fresnel media, in general heterogeneous. Their surfaces of components have the equations

$$
\begin{aligned}
q^{\prime 2} A_{m n}^{\prime} \sigma_{m}^{\prime} \sigma_{n}^{\prime}-B_{m n}^{\prime} \sigma_{m}^{\prime} \sigma_{n}^{\prime}+1 & =0, \\
q^{2} A_{m n} \sigma_{m} \sigma_{n}-B_{m n} \sigma_{m} \sigma_{n}+1 & =0,
\end{aligned}
$$$$
q^{\prime 2} \equiv \sigma_{r}^{\prime} \sigma_{r}^{\prime},
$$$$
q^{2} \equiv \sigma_{r} \sigma_{r}
$$

Since these are undegenerate central algebraic surfaces, the formula for transformation of components is homogeneous of the form (6.1). Hence we must have

$$
\begin{aligned}
B_{m n} \sigma_{m} \sigma_{n} & \equiv B_{m n}^{\prime} \sigma_{m}^{\prime} \sigma_{n}^{\prime}, \\
q^{2} A_{m n} \sigma_{m} \sigma_{n} & \equiv q^{\prime 2} A_{m n}^{\prime} \sigma_{m}^{\prime} \sigma_{n}^{\prime} .
\end{aligned}
$$


Two cases must now be distinguished, according to the way in which we break up (8.12):

\section{Case I:}

$$
q^{2} \equiv \theta^{2} q^{\prime 2}, \quad A_{m n} \sigma_{m} \sigma_{n} \equiv \theta^{-2} A_{m n}^{\prime} \sigma_{m}^{\prime} \sigma_{n}^{\prime}
$$

Case II:

$$
q^{2} \equiv \phi^{2} A_{m n} \sigma_{m}^{\prime} \sigma_{n}^{\prime}, \quad A_{m n} \sigma_{m} \sigma_{n}=\phi_{.}^{-2} q^{\prime 2} ;
$$

here $\theta, \phi$ are undetermined functions of $x_{r}$.

Let us choose a pair of corresponding points (object and image) and choose our axes in the principal directions corresponding. Then (8.11) reads

$$
\sum\left(c_{2}^{2}+c_{3}^{2}\right) \sigma_{1}^{2} \equiv \sum\left(c_{2}^{\prime 2}+c_{3}^{\prime 2}\right) \sigma_{1}^{\prime 2},
$$

where $\sum$ indicates a sum of three terms obtained by cyclic permutation of $1,2,3$. Therefore, renumbering the axes if necessary,

$$
\left\{\begin{array}{l}
\left(c_{2}^{2}+c_{3}^{2}\right) \sigma_{1}^{2} \equiv\left(c_{2}^{\prime 2}+c_{3}^{\prime 2}\right) \sigma_{1}^{\prime 2}, \\
\left(c_{3}^{2}+c_{1}^{2}\right) \sigma_{2}^{2} \equiv\left(c_{3}^{\prime 2}+c_{1}^{\prime 2}\right) \sigma_{2}^{\prime 2}, \\
\left(c_{1}^{2}+c_{2}^{2}\right) \sigma_{3}^{2} \equiv\left(c_{1}^{\prime 2}+c_{2}^{\prime 2}\right) \sigma_{3}^{\prime 2} .
\end{array}\right.
$$

Let us now consider separately Case I and Case II.

Case I: Substitution from (8.16) in (8.13) gives

$$
\begin{gathered}
\sum \frac{c_{2}^{\prime 2}+c_{3}^{\prime 2}}{c_{2}{ }^{2}+c_{3}{ }^{2}} \sigma_{1}^{\prime 2} \equiv \theta^{2} \sum \sigma_{1}^{\prime 2}, \\
\sum c_{2}{ }^{2} c_{3}{ }^{2} \frac{c_{2}^{\prime 2}+c_{3}^{\prime 2}}{c_{2}{ }^{2}+c_{3}{ }^{2}} \sigma_{1}^{\prime 2} \equiv \theta^{-2} \sum c_{2}^{\prime 2} c_{3}^{\prime 2} \sigma_{1}^{\prime 2} .
\end{gathered}
$$

From (8.17) we have

$$
\left\{\begin{array}{l}
c_{2}^{\prime 2}+c_{3}^{\prime 2}=\theta^{2}\left(c_{2}^{2}+c_{3}^{2}\right), \\
c_{3}^{\prime 2}+c_{1}^{\prime 2}=\theta^{2}\left(c_{3}^{2}+c_{1}^{2}\right), \\
c_{1}^{\prime 2}+c_{2}^{\prime 2}=\theta^{2}\left(c_{1}^{2}+c_{2}^{2}\right),
\end{array}\right.
$$

from which it follows that

$$
c_{1}^{\prime}=\theta c_{1}, \quad c_{2}^{\prime}=\theta c_{2}, \quad c_{3}^{\prime}=\theta c_{3} ;
$$

then (8.18) is automatically satisfied.

Case II: Substitution from (8.16) in (8.14) gives

$$
\sum \frac{c_{2}^{\prime 2}+c_{3}^{\prime 2}}{c_{2}^{2}+c_{3}^{2}} \sigma_{1}^{\prime 2} \equiv \phi^{2} \sum c_{2}^{\prime 2} c_{3}^{\prime 2} \sigma_{1}^{\prime 2}
$$




$$
\sum c_{2}^{2} c_{3}^{2} \frac{c_{2}^{\prime 2}+c_{3}^{\prime 2}}{c_{2}^{2}+c_{3}^{2}} \sigma_{1}^{\prime 2} \equiv \phi^{-2} \sum \sigma_{1}^{\prime 2}
$$

From (8.21) we have

$$
\left\{\begin{array}{l}
c_{2}^{\prime-2}+c_{3}^{\prime-2}=\phi^{2}\left(c_{2}^{2}+c_{3}^{2}\right), \\
c_{3}^{\prime-2}+c_{1}^{\prime-2}=\phi^{2}\left(c_{3}^{2}+c_{1}^{2}\right), \\
c_{1}^{\prime-2}+c_{2}^{\prime-2}=\phi^{2}\left(c_{1}^{2}+c_{2}^{2}\right),
\end{array}\right.
$$

and hence

$$
c_{1}^{\prime-1}=\phi c_{1}, \quad c_{2}^{\prime-1}=\phi c_{2}, \quad c_{3}^{\prime-1}=\phi c_{3} ;
$$

then (8.22) is automatically satisfied. Hence we have this result:

THEOREM III. In an absolute instrument in which the terminal media are undegenerate Fresnel media (in general heterogeneous), the principal velocities at a point in one medium are either directly or inversely proportional to the principal velocities at the corresponding point in the other medium.

9. Carathéodory's method. By (3.2), valid for an absolute instrument, we have

$$
\delta F=\sigma_{r} \delta x_{r}-\sigma_{r}^{\prime} \delta x_{r}^{\prime} .
$$

Now it is immediately obvious that if we take adjacent points $A^{\prime}, B^{\prime}$ on a ray in the initial medium in the field of an absolute instrument, their images $A, B$ lie on the final portion of the ray in question. Taking in (9.1) $\delta x_{r}^{\prime}$ to be the displacement from $A^{\prime}$ to $B^{\prime}$ and $\delta x_{r}$ to be the displacement from $A$ to $B$, and denoting the direction cosines of initial and final rays as usual by $\alpha_{r}^{\prime}, \alpha_{r}$, we may substitute in (9.1)

$$
\delta x_{r}=\alpha_{r} d s, \quad \delta x_{r}^{\prime}=\alpha_{r}^{\prime} d s^{\prime},
$$

where $d s, d s^{\prime}$ are respectively equal to $A B, A^{\prime} B^{\prime}$. Thus we obtain, on division by $d s$,

$$
\frac{\partial F}{\partial x_{r}} \alpha_{r}=\sigma_{r} \alpha_{r}-\sigma_{r}^{\prime} \alpha_{r}^{\prime} \frac{d s^{\prime}}{d s},
$$

or, by (2.1) and the homogeneity of $v$,

$$
\frac{\partial F}{\partial x_{r}} \alpha_{r}=v\left(x_{r}, \alpha_{r}\right)-v^{\prime}\left(x_{r}^{\prime}, \alpha_{r}^{\prime}\right) \frac{d s^{\prime}}{d s} .
$$

But

$$
v^{\prime}\left(x_{r}^{\prime}, \alpha_{r}^{\prime}\right) \frac{d s^{\prime}}{d s}=v^{\prime}\left(x_{r}^{\prime}, \frac{d x_{r}^{\prime}}{d s^{\prime}}\right) \frac{d s^{\prime}}{d s}=v^{\prime}\left(x_{r}^{\prime}, \frac{d x_{r}^{\prime}}{d s}\right)=v^{\prime}\left(x_{r}^{\prime}, \frac{\partial x_{r}^{\prime}}{\partial x_{s}} \alpha_{s}\right)
$$


and (9.4) thus becomes

$$
\frac{\partial F}{\partial x_{r}} \alpha_{r}=v\left(x_{r}, \alpha_{r}\right)-v^{\prime}\left(x_{r}^{\prime}, \frac{\partial x_{r}^{\prime}}{\partial x_{s}} \alpha_{s}\right) .
$$

Except for trivial changes in notation, this is the fundamental equation (14) of Carathéodory's paper (loc. cit.).

Let us now reconstruct Carathéodory's theorem regarding equality of optical lengths, filling in the part of the proof not given explicitly in his paper. We shall assume that the instrument is absolute for rays (in the field) of a certain type, corresponding to a certain one of the values $\left(v^{\prime}\right)$ of the multiplevalued medium-function of the initial medium and to a certain one of the values (v) of the multiple-valued medium-function of the final medium. We assume that these single-valued functions $v^{\prime}, v$ are even analytic functions of $\alpha_{r}^{\prime}, \alpha_{r}$ respectively, throughout three-dimensional spaces in which $\alpha_{r}^{\prime}, \alpha_{r}$ are coordinates, except perhaps for certain singular lines through the origins.

Let $P$ be any point in the region of the $\alpha_{r}$ space which is filled with lines drawn in the directions of rays lying in the field of the instrument, and let $Q$ be the reflection of $P$ in the origin of the $\alpha_{r}$ space. Let $C$ be an analytic curve joining $P$ and $Q$. Then if we put

$$
\phi(u)=\frac{\partial F}{\partial x_{r}} \alpha_{r}-\left\{v\left(x_{r}, \alpha_{r}\right)-v^{\prime}\left(x_{r}^{\prime}, \frac{\partial x_{r}^{\prime}}{\partial x_{s}} \alpha_{s}\right)\right\},
$$

where $u$ is the parameter on $C, \phi(u)$ is an analytic function which vanishes for a range of values of $u$ adjacent to $P$. Therefore $\phi(u)=0$ along $C$ and, in particular, at $Q$. But the first part of $\phi(u)$ is an odd function, and the second part is an even function. Hence, adding and subtracting the values of $\phi(u)$ at $P$ and $Q$, we get

$$
\partial F / \partial x_{r}=0, \quad v\left(x_{r}, \alpha_{r}\right)=v^{\prime}\left(x_{r}^{\prime}, \frac{\partial x_{r}^{\prime}}{\partial x_{s}} \alpha_{s}\right),
$$

throughout the field in which $P$ has been taken. Applying the analytic condition again, we see that the second relation holds for all values of $\alpha_{r}$, except for the excluded singularities. Hence if $\delta x_{r}^{\prime}, \delta x_{r}$ are object and image elements with lengths $\delta s^{\prime}, \delta s$ and direction cosines $\alpha_{r}^{\prime}, \alpha_{r}$ respectively, we have

$$
\begin{aligned}
v\left(x_{r}, \alpha_{r}\right) \delta s & =v^{\prime}\left(x_{r}^{\prime}, \frac{\partial x_{r}^{\prime}}{\partial x_{s}} \alpha_{s}\right) \delta s \\
& =v^{\prime}\left(x_{r}^{\prime}, \frac{\partial x_{r}^{\prime}}{\partial x_{s}} \delta x_{s}\right) \\
& =v^{\prime}\left(x_{r}^{\prime}, \alpha_{r}^{\prime}\right) \delta s^{\prime} .
\end{aligned}
$$


Thus Carathéodory's method establishes the equality of optical lengths for corresponding elements in an absolute instrument. The method by which the fundamental equation (9.1) is obtained in the present paper (indeed, it is deducible in a couple of lines from Hamilton's equation of the characteristic function, properly interpreted) appears more direct than the method given by Caratheodory. The latter part of the argument given above is merely the expansion of Carathéodory's proof, with a full statement of the implied conditions.

The necessary amplification of his original proof will also be found in Carathéodory, Geometrische Optik, Berlin, 1937, p. 70, which appeared after the present paper was written. Reference should be made to W. Blaschke, Abhandlungen aus dem Mathematischen Seminar der Hansischen Universität, vol. 11, 1936, pp. 409-412; although not based on the characteristic function, his argument is similar to that of the present paper, for he directs attention to the surface of components and the linear transformation (4.1). I owe this reference to W. C. Graustein.*

* The last paragraph was added in proof, May 20, 1938.

UNIVERSITY OF TORONTO,

Toronto, Canada 